\title{
Efetividade do Toque Terapêutico sobre a dor, depressão e sono em pacientes com dor crônica: ensaio clínico
}

\author{
THE EFFECTIVENESS OF THERAPEUTIC TOUCH ON PAIN, DEPRESSION AND \\ SLEEP IN PATIENTS WITH CHRONIC PAIN: CLINICAL TRIAL
}

EFECTIVIDAD DEL TOQUE TERAPÉUTICO EN DOLOR, DEPRESIÓN Y SUEÑO EN
PACIENTES CON DOLOR CRÓNICA: ENSAYO CLÍNICO

Ilda Estefani Ribeiro Marta ${ }^{1}$, Sueli Santiago Baldan², Ani Fabiana Berton ${ }^{3}$, Michele Pavam4, Maria Júlia Paes da Silva ${ }^{5}$

\section{RESUMO}

Este estudo teve como objetivo verificar a efetividade do Toque Terapêutico na diminuição da intensidade da dor, escores de auto-avaliação de depressão e melhora da qualidade do sono. Consiste em um ensaio clínico do tipo antes e depois, realizado com 30 idosos com dor crônica não-oncológica que receberam 8 sessões de Toque Terapêutico Método Krieger-Kunz na Unidade Básica de Saúde de Fernandópolis (SP), Brasil. A Escala Analógica Visual para dor foi aplicada antes e após cada sessão, o Inventário de Depressão de Beck e o Índice de Qualidade do Sono de Pittsburgh, antes da primeira e após a última. A análise dos dados demonstrou diminuição significativa $(p<0,05)$ na intensidade da dor, dos escores de auto-avaliação de depressão e do índice de qualidade do sono. Conclui-se que o Toque Terapêutico foi efetivo na diminuição da intensidade da dor, nas atitudes e nos sintomas depressivos e na melhora da qualidade do sono.

\section{DESCRITORES}

Toque terapêutico.

Terapias alternativas.

Dor.

\begin{abstract}
This research aimed to check the effectiveness of Therapeutic Touch on decreased pain intensity, depression self-assessment scores and improved sleep quality. A clinical before-after trial is presented. The study was carried out at a Basic Health Unit in Fernandópolis, SP-Brazil, involving 30 elderly patients with chronic non-oncologic pain who received 8 sessions of Therapeutic Touch in accordance with the KriegerKunz method. The Visual Analogue Scale for pain was applied before and after each session, and Beck Depression Inventory and the Pittsburgh Sleep Quality Index before the first and after the last session. Data analysis showed a significant decrease $(p<0.05)$ in pain intensity, depression selfassessment scores and the sleep quality index. It is concluded that the Therapeutic Touch was effective to decrease pain intensity and depressive attitudes and symptoms, as well as to improve sleep quality.
\end{abstract}

\section{KEY WORDS}

Therapeutic touch.

Alternative therapies. Pain

\section{RESUMEN}

Este estudio tuvo como objetivo verificar la efectividad del Tacto Terapéutico en la disminución de la intensidad del dolor, puntajes de autoevaluación de depresión y mejora de la calidad del sueño. Consistió en un ensayo clínico del tipo antes y después, realizado en una Unidad Básica de Salud de Fernandópolis - SP - Brasil, con 30 ancianos con dolor crónico no oncológico que recibieron 8 sesiones de Tacto Terapéutico Método Krieger-Kunz. La Escala Analógica Visual para dolor fue aplicada antes y después de cada sesión, el Inventario de Depresión de Beck y el Índice de Calidad del Sueño de Pittsburgh antes de la primera y luego de la última. El análisis de los datos demostró una disminución significativa $(p<$ 0,005 ) en la intensidad del dolor, de los puntajes de autoevaluación de depresión y del índice de calidad del sueño. Se concluye en que el Tacto Terapéutico fue efectivo en la disminución de la intensidad del dolor, actitudes y síntomas depresivos y en la mejora de la calidad del sueño.

\section{DESCRIPTORES}

Tacto terapéutico.

Terapias alternativas.

Dolor. ${ }^{1}$ Enfermeira. Doutora em Enfermagem Fundamental. Professora do Curso de Medicina da Universidade Camilo Castelo Branco, Campus Fernandópolis.
Fernandópolis, SP, Brasil. estefani@unicastelo.br ${ }^{2}$ Enfermeira. Especialista em Saúde da Família. Mestranda em Promoção da Saúde da Universidade de
Franca. Professora do Curso de Enfermagem das Faculdades Integradas de Fernandópolis da Fundação Educacional de Fernandópolis. Fernandópolis, SP,
Brasil. ssbaldan@fef.br ${ }^{3}$ Enfermera. Mestre em Enfermagem Psiquiátrica. Professora do Curso de Enfermagem das Faculdades Integradas de Fernandópolis
da Fundação Educacional de Fernandópolis. Fernandópolis, SP, Brasil. aniberton@fef.br ${ }^{4}$ Enfermeira Pós-Graduanda em Enfermagem do Trabalho do Centro
de Aperfeiçoamento e Pós-Graduação da Fundação Educacional de Fernandópolis. Fernandópolis, SP, Brasil. mipavam@fef.br ${ }^{5}$ Enfermeira. Professora
Titular do Departamento de Enfermagem Médico Cirúrgica da Escola de Enfermagem da Universidade de São Paulo. juliaps@usp.br

$11 \begin{aligned} & \text { Rev Esc Enferm USP } \text { Recebido: 15/03/2009 } \\ & \text { 2010; 44(4):1100-6 } \text { Aprovado: 02/04/2010 } \\ & \text { www.ee.usp.br/reeuspl } \text { Português / Inglês } \\ & \text { www.scielo.br/reeusp }\end{aligned}$




\section{INTRODUÇÃO}

O envelhecimento populacional, inicialmente ocorrido em países desenvolvidos e, mais recentemente, nos países em desenvolvimento, tornou-se um dos maiores desafios da saúde pública contemporânea ${ }^{(1)}$.

Uma das conseqüências dessa mudança no perfil demográfico é a alta incidência de doenças crônicas e degenerativas, muitas delas acompanhadas por dor e, em uma significativa parcela, por dor crônica(2)

A dor crônica é aquela que persiste além do tempo necessário para a cura de uma lesão, ou está associada a processos patológicos crônicos. Em idosos, geralmente predomina a dor do tipo crônica, relacionada à patologia degenerativa ${ }^{(3)}$.

Um estudo brasileiro com 451 idosos, servidores municipais de Londrina, PR, demonstrou que 51,4\% apresentaram queixa de dor crônica(2).

Pessoas com dor crônica, freqüentemente apresentam manifestações emocionais de depressão e alterações na qualidade do sono(3).

Dor e depressão formam uma associação complexa, que se dá em uma via de mão dupla: a dor pode gerar a depressão pela sua trajetória de perdas e incapacidades; e a depressão apresenta diferentes tipos de dor dentre seus sintomas referidos ${ }^{(4)}$.

Pesquisa realizada com 172 indivíduos de 60 anos ou mais, usuários de uma Unidade Básica de Saúde de Londrina-PR, demonstrou a incidência de dor crônica em $62,21 \%$ dos idosos estudados e, destes, 56,1\% apresentavam depressão(5).

Estudos têm demonstrado uma correlação positiva entre intensidade da dor e sintomas de ansiedade e depressão ${ }^{(6-7)}$. Enquanto a depressão, frequentemente, acompanha os quadros de dores crônicas, a ansiedade acompanha os quadros de dor aguda, ou de dores crônicas, nos quais o paciente não tem informações sobre seu estado e o que pode vir a acontecer ${ }^{(4)}$.

Alterações na qualidade do sono em pessoas com dor têm sido relatadas em alguns estudos. Em um estudo com 92 pacientes oncológicos, observou-se que sinais e sintomas somáticos, dentre eles, alterações do sono, comuns à síndrome depressiva a ao câncer, apresentaram-se com freqüência muito maior no grupo de doentes com dor do que nos sem dor ${ }^{(7)}$. Um estudo sobre a qualidade do sono em pacientes idosos com patologias vasculares periféricas demonstrou que a latência prolongada para o início do sono, embora não seja a mais comum entre idosos em geral, foi relevante nos pacientes com dor crônica ${ }^{(8)}$.

O padrão do sono e a prevalência de ansiedade e depressão foram avaliados em um estudo com 400 pessoas com dor crônica, com média de idade de 45,6 anos, com desvio padrão de 11,4 anos. A prevalência de sintomas de ansiedade foi de $72,8 \%$, de depressão $61,5 \%$ e de alteração do sono $93 \%{ }^{(9)}$.

A dor crônica afeta globalmente o indivíduo, gerando limitações e desequilíbrios biopsicossociais ${ }^{(4)}$. Sendo considerada como uma experiência que envolve o indivíduo como um todo, é compreensível que a dor possa responder a uma variedade de tratamentos e a uma combinação de diferentes modalidades terapêuticas ${ }^{(3)}$.

Uma modalidade que tem sido utilizada objetivando o alívio da dor é o Toque Terapêutico. Essa técnica está fundamentada em uma visão holística do ser humano, característica essa que motivou-nos para o estudo de sua efetividade no cuidar de pessoas idosas com dor crônica não-oncológica.

O Toque Terapêutico é baseado no uso consciente das mãos para harmonizar o campo de energia do cliente, pode ser considerado como uma interpretação contemporânea de antigas práticas de cura. A técnica do Toque Terapêutico, em geral, é dividida em quatro fases: a centralização da consciência, a avaliação do campo de energia do paciente, o reequilíbrio ou repadronização da energia e a reavaliação do campo de energia do paciente ${ }^{(10)}$.

A fase de centralização consiste no direcionamento da atenção do terapeuta para seu interior, o que lhe permite ir além do estímulo diário e das reações das interações físicas ao ambiente. Esta concentração deve ser livre de tensão e mantida durante toda a sessão de Toque Terapêutico. No cotidiano, os praticantes utilizam exercícios de respiração e visualização, dentre outros, para conseguirem centralizar suas mentes ${ }^{(10)}$.

Para a realização da fase de avaliação do campo de energia, o terapeuta movimenta suas mãos a aproximadamente cinco centímetros da pele do cliente, iniciando com as duas mãos perto da coluna dorsal, afastando as mãos, ao mesmo tempo, em direção à periferia do campo de energia. A direção céfalo-caudal pode ser seguida na avaliação posterior e anterior ${ }^{(10)}$.

Conforme percorre o campo de energia do cliente o terapeuta pode ter sensações em suas mãos, dentre elas, calor ou frio, congestão, formigamento e pequenos choques. Estas sensações geralmente indicam desequilíbrio no campo energético.

A fase de reequilíbrio ou repadronização de energia é realizada a partir do direcionamento, modulação e/ou mudança de padrões no campo de energia humano. É indicado, como exemplo, o direcionamento de energia para a área das suprarenais, em casos de clientes apresentando fadiga ou exaustão. Para obter a modulação de energia é sugerida a visualização de cores. A mudança de padrão faz-se necessária quando o campo de energia é percebido pelo terapeuta como áspero, emaranhado e inerte, dentre outros, a mudança pode ser conseguida a partir do alisamento (unruffling) do campo de energia. Para alisar determinada área do campo, o terapeuta deve mover as suas mãos, ao mesmo tempo, em direção à 
periferia do campo, repetindo este movimento três ou quatro vezes. Na quarta fase o terapeuta reavalia o campo de energia do cliente, percebendo a permanência ou desaparecimento das sensações presentes na segunda fase $\mathrm{e}^{(10)}$.

Alguns estudos realizados até o momento demonstram que o Toque Terapêutico promove diminuição da dor.

Uma revisão da literatura sobre os efeitos do Toque Terapêutico na redução da dor foi realizada incluindo estudos de 1997 a 2007, publicados em inglês. Foram encontrados nove estudos, entre 1997 e 2004, dois eram metaanálises, portanto a revisão foi realizada com cinco artigos, sendo três ensaios clínicos randomizados, um estudo longitudinal e um quasi-experimento. Quatro desses estudos demonstraram a efetividade do Toque Terapêutico na redução da dor, em especial, em casos de osteoartrites, dores músculo-esqueléticas e queimaduras ${ }^{(11)}$.

A autora da revisão citada aponta a homogeneidade e conveniência das amostras como limitações dos estudos revisados. Recomenda futuras pesquisas com amostras probabilísticas; inclusão de pessoas de diferentes etnias; duplicação de estudos para confirmação de efeitos; pesquisas sobre a duração e frequência do tratamento, necessárias para ser efetivo em diferentes tipos de dor; duração dos efeitos pós tratamento e exploração dos mecanismos e modos de ação do Toque Terapêutico(11).

Uma revisão de estudos sobre Healing Touch, terapia também baseada no uso das mãos para equilibrar o campo de energia humano, analisou pesquisas enfocando a dor, saúde mental e idosos, dentre outros. Em relação à dor, de nove estudos, sete indicaram diminuição da dor. Concluiuse que, apesar das limitações pelo pequeno tamanho das amostras, variedade de instrumentos de medida e diferentes condições de dor, os resultados encorajam o uso dessa técnica no manejo da dor ${ }^{(12)}$.

Nessa mesma revisão foram analisados cinco estudos tendo idosos como sujeitos; foi demonstrada diminuição da dor, de agitação em casos de demência, melhora do apetite, do sono e socialização, dentre outros. No entanto, os estudos também foram limitados pelo pequeno tamanho das amostras ${ }^{(12)}$.

Da mesma forma, os estudos de efetividade do Healing Touch em saúde mental, não permitiram conclusões adequadas, devido ao tamanho da amostra e controles inadequados. Os autores dessa revisão enfatizam que, apesar de muitos resultados positivos serem relatados, tais como redução do estresse, ansiedade e dor, nenhum achado foi conclusivo. Muitos estudos careciam de informações importantes, principalmente no que se refere à validade interna e externa. Defendem a necessidade de novas pesquisas e de replicações das pesquisas já existentes para testar o efeito potencial do Healing Touch, uma vez que acreditam que essa técnica pode se constituir em outra opção para enfermeiros no cuidado seguro e não invasivo ${ }^{(12)}$.

Apesar das limitações dos estudos, a efetividade do Toque Terapêutico na diminuição da dor tem sido demons- trada por alguns estudos bem delineados, no entanto, sua efetividade sobre depressão e qualidade do sono é pouco explorada na literatura.

Uma pesquisa brasileira demonstrou que a aplicação do Toque Terapêutico em pessoas com dor crônica não-oncológica promoveu diminuição significativa da intensidade da dor e escores de auto-avaliação de depressão e aumento da positividade do significado psicológico da dor. Ainda nesta pesquisa, embora não constasse dos objetivos, alguns sujeitos relataram espontaneamente que estavam dormindo melhor ${ }^{(13)}$.

Considerando a epidemiologia da dor crônica em idosos e a coexistência desta com a depressão e alterações do sono torna-se pertinente empregar esforços na investigação do Toque Terapêutico como possibilidade de intervenção para a melhora dessas condições.

\section{OBJETIVO}

Avaliar a efetividade do Toque Terapêutico na diminuição da intensidade da dor, escores de auto-avaliação de depressão e melhora da qualidade do sono em pessoas com dor crônica não-oncológica.

\section{MÉTODO}

Foi realizado um ensaio clínico do tipo antes e depois, na Unidade de Saúde da Família Américo Possari, Bairro Paraíso, cidade de Fernandópolis, noroeste paulista.

A amostra foi constituída por 30 pessoas, com idade igual ou superior a 60 anos, usuárias da unidade referida.

A seleção da amostra foi aleatória, inicialmente foi realizado um levantamento do total de idosos usuários da unidade. A partir de visitas domiciliarias foram identificados os idosos com dor crônica e, dentre esses, foi realizado um sorteio para determinar a amostra.

Para inclusão na amostra utilizou-se como critério a presença de dor não-oncológica por um período superior a 6 (seis) meses; persistência da dor apesar da terapêutica médica; disponibilidade para comparecer à unidade de saúde duas vezes por semana, durante quatro semanas; período mínimo de seis horas entre a última dose de medicação analgésica e a sessão de Toque Terapêutico (controlado a cada sessão) e possibilidade de manutenção da terapêutica anterior durante o período de coleta de dados.

Após aprovação do projeto pelo CEP da EEUSP (Processo $n$ - 662/2007), os sujeitos foram contatados nos domicílios ou na unidade de saúde. Seguido da explicação detaIhada dos objetivos e métodos da pesquisa, pedimos que assinassem o Termo de Consentimento Livre e Esclarecido e preenchemos uma ficha de caracterização do sujeito.

Cada sujeito recebeu 8 (oito) sessões de Toque Terapêutico Método Krieger-Kunz, durante 1(um) mês, sendo 2 (duas) por semana, às segundas e quartas-feiras para alguns dos sujeitos e às terças e quintas-feiras para os demais. As sessões 
foram realizadas individualmente, em salas da unidade de saúde, por três das pesquisadoras com treinamento prévio na técnica. A duração de cada sessão foi em torno de 25 minutos.

Para avaliação da dor foi utilizada a Escala Analógica Visual (VAS), aplicada antes e após cada sessão. Essa escala é unidimensional e permite quantificar a intensidade da dor. Nessa pesquisa, a escala foi apresentada como uma linha reta, horizontal, de 10 (dez) centímetros, não numerada, constando na extremidade esquerda as palavras sem dor e na extremidade direita pior dor possível. Os sujeitos foram instruídos a marcar um ponto nessa linha, correspondente à intensidade da dor que estavam sentindo no momento. $\mathrm{O}$ escore foi obtido medindo-se, com régua, a distância entre o ponto sem dor, correspondendo ao valor 0 (zero) e a marca feita pelo sujeito. Dessa forma, os valores da escala variaram de 0 (zero) a 10 (dez).

Para auto-avaliação da depressão foi aplicado o Inventário de Depressão de Beck (BDI), antes da primeira e após a última sessão. Esse instrumento, já validado no Brasil ${ }^{(14)}$, é apresentado em forma de auto-relato e permite uma avaliação quantitativa da intensidade dos sintomas depressivos. Em sua forma original, usada nessa pesquisa, é composto por 21 categorias de sintomas e atitudes; cada categoria consiste em uma série graduada de 4 (quatro) afirmações, para cada afirmação é atribuído um valor de 0 (zero) a 3 (três), indicando o grau de severidade dos sintomas. A escolha do ponto de corte desse inventário deve variar de acordo com a natureza da amostra e os objetivos do pesquisador ${ }^{(15-16)}$. Tendo em vista que um dos objetivos do presente estudo foi verificar o efeito do Toque Terapêutico sobre os escores de auto-avaliação de depressão, não foi feita uma identificação de sujeitos deprimidos e não deprimidos.

A qualidade do sono foi medida antes da primeira e após a última sessão de Toque Terapêutico, a partir da utilização do Índice de Qualidade do Sono de Pittsburgh (PSQI) ${ }^{(17)}$, também já validado para a cultura brasileira ${ }^{(18)}$. Para responder às questões, os sujeitos foram instruídos a considerarem os acontecimentos no período de 1 (um) mês anterior à data de coleta de dados. Esse instrumento é constituído por 7 (sete) componentes com pontuação de 0 (zero) a 3 (três), de forma que a pontuação máxima é de 21 pontos. Um escore global superior a 5 (cinco) indica má qualidade do sono ${ }^{(17)}$.

Considerando a baixa escolaridade e idade da amostra do presente estudo, as afirmações do Inventário de Depressão de Beck e as questões do Índice de Qualidade do Sono de Pittsburgh foram lidas, de forma lenta e em tom de voz audível; assim que o sujeito fazia sua escolha, a mesma era registrada por uma das pesquisadoras.

Foi realizado um teste das diferenças entre as médias dos sujeitos antes e depois de cada sessão de Toque Terapêutico para a intensidade da dor; e antes da primeira e depois da última sessão para os escores de auto-avaliação de depressão e qualidade do sono. Em todos os casos foi utilizado o teste $t$ de Student pareado e foram considerados significativos os valores de $p<0,05$. 0 estudo tem delineamento antes-depois, de forma que o sujeito foi seu próprio controle.

\section{RESULTADOS}

\section{Caracterização da amostra}

Dos 30 sujeitos que participaram desse estudo, $80 \%$ são do sexo feminino e $20 \%$ masculino, com faixa etária variando entre 60 e 80 anos. Em relação ao estado civil, 66,67\% são casados, $30 \%$ viúvos e 3,33\% separados.

Quanto à escolaridade, 23,33\% são analfabetos, 70\% cursaram o ensino fundamental de forma incompleta e $6,67 \%$ o fundamental completo.

Do total de sujeitos, 1 (3,33\%) ainda exerce atividade de comerciante, 5 senhoras ( $16,67 \%$ ) cuidam dos afazeres domésticos e 24 (80\%) são aposentados ou pensionistas.

Em relação à religião, $80 \%$ se declaram católicos, 13,33\% evangélicos e 6,67\% espíritas.

Com referência à localização da dor, 33,34\% apontaram a coluna vertebral, $23,34 \%$ os membros inferiores, $13,33 \%$ os membros superiores, $13,33 \%$ coluna vertebral e membros inferiores, $10 \%$ coluna vertebral e membros superiores, 3,33\% membros superiores e inferiores e 3,33\% coluna vertebral e cabeça. $O$ tempo de existência da dor variou de 8 (oito) meses a 40 anos.

\section{Avaliação da intensidade da dor antes e depois das sessões de Toque Terapêutico}

Considerando cada sessão individualmente, os valores apresentados na Tabela 1 mostram uma redução significativa da intensidade da dor em todas as sessões $(p<0,05)$.

O teste estatístico da diferença entre as médias dos escores da VAS antes da primeira $(1,647)$ e após a oitava sessão $(0,270)$ também mostrou diminuição significativa $(p=$ $0,007)$ da intensidade da dor.

Em termos percentuais, quando consideramos a média dos escores da VAS antes da primeira e após a última sessão de Toque Terapêutico, houve $83,61 \%$ de redução na intensidade da dor.

Tabela 1 - Diferença entre as médias da Escala Analógica Visual para dor antes e depois de cada sessão de Toque Terapêutico Fernandópolis - 2009

\begin{tabular}{lccc}
\hline \multirow{2}{*}{ Sessão } & Antes da Sessão & Depois da Sessão & \multirow{2}{*}{ Valor de p } \\
\cline { 2 - 3 } & Média \pm DP & Média \pm DP & \\
\hline Primeira & $1,647 \pm 2,469$ & $0,867 \pm 1,793$ & 0,032 \\
Segunda & $2,520 \pm 3,121$ & $1,203 \pm 2,140$ & 0,000 \\
Terceira & $2,370 \pm 2,516$ & $0,947 \pm 1,485$ & 0,002 \\
Quarta & $1,713 \pm 2,087$ & $0,547 \pm 0,975$ & 0,001 \\
Quinta & $1,010 \pm 1,499$ & $0,417 \pm 1,047$ & 0,001 \\
Sexta & $1,773 \pm 2,227$ & $0,367 \pm 0,756$ & 0,001 \\
Sétima & $1,020 \pm 1,583$ & $0,293 \pm 0,641$ & 0,002 \\
Oitava & $0,893 \pm 1,691$ & $0,270 \pm 1,102$ & 0,001 \\
\hline
\end{tabular}

Nota: $(\mathrm{N}=30)$ 


\section{Escores de auto-avaliação de depressão antes e depois do Toque Terapêutico}

A análise das médias em cada uma das categorias mostrou diminuição significativa nas categorias humor, desejo de auto-punição, isolamento social, inibição para o trabalho, distúrbios do sono e fadiga, conforme observado na Tabela 2.

Tabela 2 - Diferença entre as médias do Inventário de Depressão de Beck, por categoria, antes da primeira e após a última sessão de Toque Terapêutico - Fernandópolis - 2009

\begin{tabular}{|c|c|c|c|}
\hline Categorias & $\begin{array}{l}\text { Antes da Primeira } \\
\text { Sessão Média } \pm \text { DP }\end{array}$ & $\begin{array}{l}\text { Depois da Última } \\
\text { Sessão Média } \pm \text { DP }\end{array}$ & $\begin{array}{l}\text { Valor } \\
\text { de p }\end{array}$ \\
\hline 1 Humor & $1,833 \pm 0,985$ & $1,133 \pm 0,345$ & 0,000 \\
\hline 2 Pessimismo & $1,533 \pm 0,899$ & $1,200 \pm 0,484$ & 0,057 \\
\hline $\begin{array}{l}3 \text { Sensação de } \\
\text { Fracasso }\end{array}$ & $1,300 \pm 0,749$ & $1,066 \pm 0,253$ & 0,090 \\
\hline 4 Insatisfação & $1,100 \pm 0,305$ & $1,200 \pm 0,484$ & 0,326 \\
\hline $\begin{array}{l}5 \text { Sensação de } \\
\text { Culpa }\end{array}$ & $1,366 \pm 0,927$ & $1,200 \pm 0,664$ & 0,455 \\
\hline $\begin{array}{l}6 \text { Sentimento de } \\
\text { Punição }\end{array}$ & $1,433 \pm 1,040$ & $1,300 \pm 0,794$ & 0,459 \\
\hline 7 Ódio a si mesmo & $1,266 \pm 0,639$ & $1,100 \pm 0,305$ & 0,134 \\
\hline 8 Auto Acusação & $1,266 \pm 0,691$ & $1,166 \pm 0,461$ & 0,541 \\
\hline $\begin{array}{l}9 \text { Desejo de } \\
\text { Auto Punição }\end{array}$ & $1,166 \pm 0,379$ & $1,000 \pm 0,000$ & 0,023 \\
\hline $\begin{array}{l}10 \text { Período } \\
\text { de Choro }\end{array}$ & $1,633 \pm 0,889$ & $1,233 \pm 0,773$ & 0,097 \\
\hline 11 Irritabilidade & $1,533 \pm 0,681$ & $1,266 \pm 0,639$ & 0,118 \\
\hline $\begin{array}{l}12 \text { Isolamento } \\
\text { Social }\end{array}$ & $1,366 \pm 0,850$ & $1,033 \pm 0,182$ & 0,023 \\
\hline 13 Indecisão & $1,566 \pm 0,897$ & $1,233 \pm 0,678$ & 0,096 \\
\hline 14 Imagem Corporal & $1,266 \pm 0,639$ & $1,233 \pm 0,678$ & 0,787 \\
\hline $\begin{array}{l}15 \text { Inibição para } \\
\text { o Trabalho }\end{array}$ & $1,666 \pm 0,802$ & $1,266 \pm 0,449$ & 0,008 \\
\hline $\begin{array}{l}16 \text { Distúrbios } \\
\text { do Sono }\end{array}$ & $2,033 \pm 1,033$ & $1,433 \pm 0,935$ & 0,010 \\
\hline 17 Fadiga & $1,933 \pm 1,048$ & $1,400 \pm 0,621$ & 0,013 \\
\hline $\begin{array}{l}18 \text { Perda de } \\
\text { Apetite }\end{array}$ & $1,466 \pm 0,776$ & $1,333 \pm 0,711$ & 0,255 \\
\hline 19 Perda de Peso & $1,233 \pm 0,504$ & $1,300 \pm 0,702$ & 0,489 \\
\hline $\begin{array}{l}20 \text { Preocupação } \\
\text { Somática }\end{array}$ & $1,566 \pm 0,858$ & $1,433 \pm 0,626$ & 0,380 \\
\hline 21 Perda da Libido & $1,866 \pm 1,166$ & $1,600 \pm 1,101$ & 0,174 \\
\hline
\end{tabular}

Nota: $(\mathrm{N}=30)$

Antes da primeira sessão de Toque Terapêutico, o distúrbio do sono foi a categoria com maior valor de média na auto-avaliação de depressão, seguida pelas categorias fadiga, perda da libido, humor e inibição para o trabalho. Excetuando a categoria perda da libido, todas apresentaram diminuição significativa da média após a última sessão.

A média global dos escores do Inventário de Depressão de Beck antes do Toque Terapêutico foi de 31,43 e depois da última sessão foi de 26,60. O teste estatístico mostrou uma diferença significativa entre as médias $(p=0,006)$.

Em termos percentuais houve redução de 15,37\% no escore geral de auto-avaliação de depressão.

\section{Escores da qualidade do sono antes e depois do Toque Terapêutico}

Considerando cada componente individualmente, conforme observado na Tabela 3, houve redução estatisticamente significativa nos escores da latência do sono, duração do sono, eficiência habitual do sono, distúrbios do sono e sonolência diurna e distúrbios durante o dia.

Tabela 3 - Diferença entre as médias do Índice de Qualidade do Sono de Pittsburgh, por componente, antes da primeira e depois da última sessão de Toque Terapêutico - Fernandópolis - 2009

\begin{tabular}{lccc}
\hline Componentes & $\begin{array}{c}\text { Antes da Primeira } \\
\text { Sessão Média } \pm \text { DP }\end{array}$ & $\begin{array}{c}\text { Depois da Última } \\
\text { Sessão Média } \pm \text { DP }\end{array}$ & $\begin{array}{c}\text { Valor } \\
\text { de p }\end{array}$ \\
\hline $\begin{array}{l}\text { 1 Qualidade } \\
\text { subjetiva do sono } \\
\text { 2 Latência } \\
\text { do sono }\end{array}$ & $1,166 \pm 0,698$ & $0,866 \pm 0,507$ & 0,059 \\
$\begin{array}{l}\text { 3 Duração } \\
\text { do sono }\end{array}$ & $1,633 \pm 0,949 \pm 1,220$ & $1,000 \pm 1,144$ & 0,008 \\
$\begin{array}{l}\text { 4 Eficiência } \\
\text { habitual do sono }\end{array}$ & $0,866 \pm 1,136$ & $0,333 \pm 0,844$ & 0,021 \\
$\begin{array}{l}\text { 5 Distúrbio } \\
\text { do sono }\end{array}$ & $1,766 \pm 0,626$ & $1,233 \pm 0,504$ & 0,000 \\
$\begin{array}{l}\text { 6 Uso de } \\
\text { medicamentos }\end{array}$ & $0,933 \pm 1,311$ & $0,533 \pm 1,136$ & 0,070 \\
$\begin{array}{l}\text { 7 Sonolência diurna } \\
\text { e Distúrbios durante } \\
\text { o dia }\end{array}$ & $1,033 \pm 1,066$ & $0,333 \pm 0,479$ & 0,002 \\
\hline $\begin{array}{l}\text { Nota: (N=30) } \\
\text { no }\end{array}$ & & & \\
\hline
\end{tabular}

A média global dos escores de qualidade do sono, antes da primeira sessão de Toque Terapêutico foi 9,067 e após a última sessão 5,233. A diferença entre essas médias foi significativa $(p=0,000)$.

Houve, em termos percentuais, uma redução de 42,29\% no escore global após 8 (oito) sessões de Toque Terapêutico.

\section{DISCUSSÃO}

Os resultados dessa pesquisa sugerem que o Toque Terapêutico reduziu significativamente a intensidade da dor crônica na amostra estudada. Esse dado é consistente com os achados da literatura sobre essa temática.

Um estudo com 95 idosos, randomizados em grupo experimental, grupo placebo e grupo de cuidados de rotina, testou o efeito do Toque Terapêutico sobre a dor crônica, ansiedade e cortisol salivar, esse último como indicador fisiológico de estresse. O grupo experimental apresentou redução significativa da dor e ansiedade, o nível de cortisol salivar não apresentou diferença significativa. Concluiu-se que o Toque Terapêutico foi efetivo na redução da dor e ansiedade em população idosa ${ }^{(19)}$.

A redução da dor aguda também vem sendo demonstrada em estudos enfocando a efetividade do Toque Terapêutico. $O$ efeito do Toque Terapêutico sobre a dor pós-
Efetividade do Toque Terapêutico sobre a dor, depressão e sono em pacientes com dor crônica: ensaio clínico Marta IER, Baldan SS, Berton AF, Pavam M, Silva MJP 
cirúrgica foi estudado em uma amostra de 90 idosos, divididos em grupo experimental, controle e placebo. Os resultados demonstraram que, no grupo experimental, houve diminuição estatisticamente significativa na intensidade da dor quando comparado aos demais grupos. A freqüência de pulso e tamanho das pupilas, usados como medidas de relaxamento, não apresentaram diferenças significativas entre os grupos ${ }^{(20)}$.

Os resultados do presente estudo indicam também que o Toque Terapêutico foi efetivo na redução dos escores de auto-avaliação de depressão da amostra estudada. Esse achado reveste-se de especial importância, tendo em vista a escassez de estudos sobre a efetividade do Toque Terapêutico na depressão.

Um quasi-experimento foi realizado com 30 pessoas com depressão moderada a severa, divididos em grupo experimental e grupo de tratamento de rotina; o grupo experimental recebeu 6 (seis) sessões de Healing Touch, sendo 2 (duas) por semana e teve redução estatisticamente significativa dos escores de auto-avaliação de depressão, avaliados pelo Inventário de Depressão de Beck ${ }^{(21)}$.

Outro estudo quasi-experimental, com delineamento antes-depois, teve como amostra 30 pessoas com dor crônica não-oncológica, com idade variando entre 34 a 79 anos, que receberam 2 (duas) sessões semanais de Toque Terapêutico, por 4 (quatro) semanas. O Inventário de Depressão de Beck foi aplicado antes da primeira e após a última sessão e a média do escore global apresentou diminuição significativa. Quando considerado o escore de cada uma das 21 categorias, os resultados demonstraram diminuição significativa do ponto de vista estatístico no humor, ódio a si mesmo, auto-acusação, inibição para o trabalho, distúrbios do sono, fadiga, perda de peso, preocupação somática e perda da libido ${ }^{(13)}$.

No presente estudo houve diminuição significativa nas categorias humor, desejo de auto-punição, isolamento social, inibição para o trabalho, distúrbios do sono e fadiga.

A análise dos dados do presente estudo sugere, ainda, que o Toque Terapêutico foi efetivo na melhora da qualidade do sono.

Embora nenhuma pesquisa objetivando avaliar a efetividade do Toque Terapêutico sobre a qualidade do sono tenha sido encontrada, podemos pensar que a diminuição da intensidade da dor contribui para a melhora da qualidade do sono. Nesse aspecto enfatizamos o estudo, já citado anteriormente, no qual as alterações do sono foram muito mais freqüentes no grupo de doentes com câncer que apresentavam dor do que nos doentes sem dor ${ }^{(7)}$.

Outro artigo, também citado anteriormente, demonstrou que a latência prolongada para o início do sono foi relevante nos pacientes com dor crônica $^{(8)}$. No presente estudo, a latência do sono foi o componente com maior valor de média antes das sessões de Toque Terapêutico e teve diminuição significativa após a última sessão.

Diante dos resultados desse estudo, gostaríamos de salientar o caráter de terapia complementar do Toque Terapêutico, uma vez que sua utilização não se propõe a substituir outras formas de tratamento.

Embora nas publicações sobre Toque Terapêutico seja utilizado o termo heal, buscamos transcender a tradução literal deste termo, associando-o à qualidade de vida, mudanças internas e transcendência das condições de vida(10). Os praticantes de Toque Terapêutico não almejam, portanto, promover a cura no sentido usual da área médica; estão interessados em ajudar o cliente a atingir um estado de bem-estar.

Enquanto estratégia de cuidar em enfermagem, a prática do Toque Terapêutico conta com o aval do Conselho Federal de Enfermagem, conforme a resolução COFEN-197/ 1997, que estabelece e reconhece as terapias complementares como especialidade e/ou qualificação do profissional de Enfermagem ${ }^{(22)}$.

Ressaltamos algumas limitações desse estudo, dentre elas, o fato de se constituir em um ensaio clínico, tipo antes e depois e não um ensaio clínico randomizado e controlado, que teria maior nível de evidência. O delineamento desse estudo também não permite a generalização dos resultados obtidos.

Apesar das limitações consideramos que o presente estudo propicia conclusões favoráveis à prática do Toque Terapêutico no cuidado de idosos com dor crônica nãooncológica.

\section{CONCLUSÃO}

O Toque Terapêutico foi efetivo na diminuição da intensidade da dor e das atitudes e sintomas depressivos, assim como na melhora da qualidade do sono na amostra estudada.

Os resultados sugerem que o Toque Terapêutico, enquanto uma modalidade complementar, não invasiva, pode beneficiar idosos com dor crônica, sintomas e atitudes depressivas e má qualidade do sono. Não obstante, faz-se necessária a realização de novas investigações que possam superar as limitações encontradas na maioria das publicações dessa área e produzir conhecimentos relativos à efetividade do Toque Terapêutico na melhora da qualidade do sono e depressão.

Acreditamos que a prática do Toque Terapêutico atende aos anseios de uma atuação holística, baseada na visão integral do ser humano, comumente relatada na literatura de enfermagem. 


\section{REFERÊNCIAS}

1. Lima-Costa MF, Veras R. Saúde pública e envelhecimento. Cad Saúde Pública. 2003;19(3): 700-1.

2. Dellaroza MSG, Pimenta CAM, Matsuo T. Prevalência e caracterização da dor crônica em idosos não institucionalizados. Cad Saúde Pública. 2007;23(5):1151-60.

3. Figueiró JA. A dor. São Paulo: Publifolha; 2000.

4. Baptista CMA, Ebel R, Fortes S. Grupoterapia e dor crônica. In: Figueiro JAB, Angelotti G, Pimenta CAM, organizadores. Dor e saúde mental. São Paulo: Atheneu; 2005. p. 167-79.

5. Dellaroza MSG, Furuya RK, Cabrera MAS, Matsuo T, Trelha C, Yamada KN, et al. Caracterização da dor crônica e métodos analgésicos utilizados por idosos na comunidade. Rev Assoc Med Bras. 2008;54(1):36-41.

6. Brasil ISPS, Ponde MP. Sintomas ansiosos e depressivos e sua correlação com intensidade da dor em pacientes com neuropatia periférica. Rev Psiquiatr. 2009;31(1):24-31.

7. Pimenta CAM, Koizumi MS, Teixeira MJ. Dor crônica e depressão: estudo em 92 doentes. Rev Esc Enferm USP. 2000;34(1):76-83.

8. Corrêa K, Ceolim MF. Qualidade do sono em pacientes idosos com patologias vasculares periféricas. Rev Esc Enferm USP. 2008;42(1):12-8.

9. Castro MMC, Daltro C. Sleep patterns and symptoms of anxiety and depression in patients with chronic pain. Arq Neuropsiquiatr. 2009;67(1):25-8.

10. Krieger D. O toque terapêutico: versão moderna da antiga técnica de imposição de mãos. São Paulo: Cultrix; 1995.

11. Monroe CM. The effects of therapeutic touch on pain. J Holist Nurs. 2009;27(2):85-9.

12. Wardell DW, Weymouth KF. Review of studies of healing touch. J Nurs Scholarsh. 2004; 36(2):147-54.
13. Marta IER. Utilização do toque terapêutico no processo de cuidar de pessoas com dor crônica não-neoplásica: avaliação da experiência pelo paciente e verificação dos efeitos sobre a intensidade, significado psicológico da dor e escores de autoavaliação de depressão [tese]. Ribeirão Preto: Escola de Enfermagem de Ribeirão Preto, Universidade de São Paulo; 1999.

14. Gorenstein C, Andrade L. Inventário de depressão de Beck: propriedades psicométricas da versão em português. Rev Psiq Clin. 1998;25(2):245-50.

15. Beck AT, Ward CH, Mendelson M, Mock J, Erbaugh J. An inventory for measuring depression. Arch Gen Psychiatry. 1961;4:561-71.

16. Beck AT, Steer RA, Garbin MG. Psychometric properties of the Beck Depression Inventory: twenty-five years of evaluation. Clin Psychol Rev. 1988;8(1):77-100.

17. Buysse DJ, Reynolds III CF, Monk TH, Berman SR, Kupfer DJ. The Pittsburgh Sleep Quality Index: a new instrument for psychiatric practice and research. Psychiatry Res. 1989; 28(2):193-213.

18. Ceolim MF. Padrões de atividades e de fragmentação do sono em pessoas idosas [tese]. Ribeirão Preto: Escola de Enfermagem de Ribeirão Preto, Universidade de São Paulo; 1999.

19. Lin YS, Taylor AG. Effects of therapeutic touch in reducing pain and anxiety in an elderly population. Integr Med. 1998;1(4):155-62.

20. McCormack GL. Using non-contact therapeutic touch to manage post-surgical pain in the elderly. Occup Ther Int. 2009;16(1):44-56.

21. Bradway C. Effects of healing touch on depression. Healing Touch Newsl. 1998;8(3):2.

22. Conselho Federal de Enfermagem. Resolução COFEN-197/ 1997. Estabelece e reconhece as Terapias Alternativas como especialidade e/ou qualificação do profissional de Enfermagem [legislação na Internet]. Rio de Janeiro; 1997. [citado 2009 jan. 23]. Disponível em: http://www.portalcofen.gov.br/Site/ 2007/materias. asp?Article $I D=7041$ \&section $\mid D=34$
Correspondência: Ilda Estefani Ribeiro

Rua Aviador Eduardo Borges de Freitas, 157 - Residencial Terra Verde CEP 15600-000 - Fernandópolis, SP, Brasil 